\title{
Dissecting the default mode network: direct structural evidence on the morphology and axonal connectivity of the fifth component of the cingulum bundle
}

\author{
Georgios P. Skandalakis, MD, MSc, ${ }^{1-3,10}$ Spyridon Komaitis, MD, MSc, ${ }^{1,2,4}$ \\ Aristotelis Kalyvas, MD, MSc, ${ }^{1-3,5}$ Evgenia Lani, MD, ${ }^{1-3}$ Chrysoula Kontrafouri, MD, ${ }^{1-3}$ \\ Evangelos Drosos, MD, ${ }^{1,2}$ Faidon Liakos, MD, 1,3 Maria Piagkou, MD, PhD, 1,3 \\ Dimitris G. Placantonakis, MD, PhD, ${ }^{6}$ John G. Golfinos, MD, ${ }^{6}$ Kostas N. Fountas, MD, PhD, ${ }^{8}$ \\ Eftychia Z. Kapsalaki, MD, PhD, ${ }^{7}$ Constantinos G. Hadjipanayis, MD, PhD,, 910 \\ George Stranjalis, MD, PhD, ${ }^{1,2,4}$ and Christos Koutsarnakis, MD, MSc ${ }^{1-4}$
}

\begin{abstract}
${ }^{1}$ Athens Microneurosurgery Laboratory, Evangelismos Hospital, Athens; ${ }^{2}$ Department of Neurosurgery, National and Kapodistrian University of Athens; ${ }^{3}$ Department of Anatomy, Medical School, National and Kapodistrian University of Athens; ${ }^{4}$ Hellenic Center for Neurosurgical Research, "Petros Kokkalis," Athens, Greece; '5epartment of Neurosurgery, Toronto Western Hospital, University Health Network, Toronto, Ontario, Canada; ${ }^{\circ}$ Department of Neurosurgery, NYU School of Medicine, New York, New York; Departments of ${ }^{7}$ Radiology and ${ }^{8}$ Neurosurgery, School of Medicine, University of Thessaly, Larisa, Greece; ${ }^{9}$ Department of Neurosurgery, Mount Sinai Union Square, New York; and ${ }^{10}$ Department of Neurosurgery, Icahn School of Medicine at Mount Sinai, New York, New York
\end{abstract}

OBJECTIVE Although a growing body of data support the functional connectivity between the precuneus and the medial temporal lobe during states of resting consciousness as well as during a diverse array of higher-order functions, direct structural evidence on this subcortical circuitry is scarce. Here, the authors investigate the very existence, anatomical consistency, morphology, and spatial relationships of the cingulum bundle $\mathrm{V}$ (CB-V), a fiber tract that has been reported to reside close to the inferior arm of the cingulum (Cingl).

METHODS Fifteen normal, formalin-fixed cerebral hemispheres from adults were treated with Klingler's method and subsequently investigated through the fiber microdissection technique in a medial to lateral direction.

RESULTS A distinct group of fibers is invariably identified in the subcortical territory of the posteromedial cortex, connecting the precuneus and the medial temporal lobe. This tract follows the trajectory of the parietooccipital sulcus in a close spatial relationship with the Cingl and the sledge runner fasciculus. It extends inferiorly to the parahippocampal place area and retrosplenial complex area, followed by a lateral curve to terminate toward the fusiform face area (Brodmann area [BA] 37) and lateral piriform area (BA35). Taking into account the aforementioned subcortical architecture, the CB-V allegedly participates as a major subcortical stream within the default mode network, possibly subserving the transfer of multimodal cues relevant to visuospatial, facial, and mnemonic information to the precuneal hub. Although robust clinical evidence on the functional role of this stream is lacking, the modern neurosurgeon should be aware of this tract when manipulating cerebral areas en route to lesions residing in or around the ventricular trigone.

CONCLUSIONS Through the fiber microdissection technique, the authors were able to provide original, direct structural evidence on the existence, morphology, axonal connectivity, and correlative anatomy of what proved to be a discrete white matter pathway, previously described as the CB-V, connecting the precuneus and medial temporal lobe.

https://thejns.org/doi/abs/10.3171/2020.2.JNS193177

KEYWORDS cingulum; precuneus; temporal lobe; default mode network; brain connectivity; brain anatomy

ABBREVIATIONS $\mathrm{BA}=$ Brodmann area; $\mathrm{CB}-\mathrm{V}=$ cingulum bundle $\mathrm{V} ; \mathrm{Cingl}=$ inferior arm of the cingulum; $\mathrm{CTTP}=$ contralateral interhemispheric transfalcine transprecuneus approach; DMN = default mode network; FFA = fusiform face area; FrM = forceps major; ILF = inferior longitudinal fasciculus; POS = parietooccipital sulcus; PPA = parahippocampal place area; RSC = retrosplenial cortex; Slg = sledge runner fasciculus; STTS = supracerebellar transtentorial transcollateral sulcus. SUBMITTED November 24, 2019. ACCEPTED February 10, 2020.

INCLUDE WHEN CITING Published online April 24, 2020; DOI: 10.3171/2020.2.JNS193177. 
$\mathrm{D}$ URING the last decade, a growing body of evidence has advocated the functional connectivity of the precuneus with areas of the temporal lobe. ${ }^{1,2}$ This synergy is a basic component of the default mode network (DMN), which is activated when the brain is in the condition of "resting consciousness," and allegedly subserves high-order functions such as planning, volition, episodic memory, attention, spatial updating, error detection, social intelligence, intelligence comparison, and verbal creativity. ${ }^{3-10}$ Stronger connectivity of these regions is positively correlated with higher cognitive performance, ${ }^{11}$ while in neurological and psychiatric conditions the activation of this circuitry is markedly altered. ${ }^{12-14}$ Although the functional connectivity of the aforementioned cerebral regions and its significance have been repeatedly reported, the anatomical constraints of underlying structural circuits need to be further elucidated.

To this end, we focus on a long association fiber tract, previously described as the cingulum bundle V (CB-V), which is believed to interconnect areas of the temporal lobe and the precuneus..$^{15}$ Employing the fiber microdissection technique in formalin-fixed brains, we provide direct anatomical evidence on the tract's invariable existence, morphological silhouette, termination pattern, and spatial relationship with adjacent fiber tracts, namely the inferior arm of the cingulum (CingI), the sledge runner fasciculus (Slg), the inferior longitudinal fasciculus (ILF), and the forceps major (FrM). We present our results with the aim of refining current knowledge about and documenting structural brain connectivity and organization in terms of cortical and subcortical axonal interactions. Moreover, we provide useful insights, allowing for the study of high-level functioning circuits residing in the posteromedial cortices in greater detail and thus paving the way toward a more comprehensive understanding of these networks in both normal and pathological brain conditions. Last, we offer surgical comments and functional considerations on the involvement of the fifth subcomponent of the CB in operative trajectories designed to access lesions in and around the ventricular trigone.

\section{Methods}

Fifteen normal cerebral hemispheres were obtained from 8 different adults in routine autopsy and were fixed in a $10 \%$ formalin solution for 8 weeks. Upon meticulous dissection of the arachnoid membrane and vessels, all specimens were treated with Klingler's method and investigated through the fiber microdissection technique. The operating microscope (OPMI Plus, Carl Zeiss AG) and microsurgical instruments, such as fine metallic periosteal elevators, variously sized anatomical forceps, and microscissors, were used as previously described. ${ }^{16-19}$ Because the area of interest is located deep to the parietooccipital sulcus (POS), distal calcarine fissure, and medial temporal lobe, we focused all dissections on this cerebral territory. Prior to dissection, the regional sulcal anatomy consisting of the POS, proximal calcarine fissure, distal calcarine fissure, collateral sulcus, occipitotemporal sulcus, and subparietal sulcus was studied in all cases.

We then started the fiber dissection process by me- ticulously peeling away the cortex and underlying short association fibers - known as arcuate, or U, fibers - at the level of the POS and up to the distal end of the calcarine sulcus. Cortical and white matter fiber dissections gradually proceeded to include the cuneus, precuneus, posterior cingulate cortex, retrosplenial cortex (RSC), ventral and medial temporal lobe, and basal temporooccipital area in an effort to map and record the topography, morphology, termination pattern, and correlative anatomy of the CB-V.

Digital photographs were obtained in a stepwise fashion at different dissection stages and angles to illustrate more vividly the topographical architecture and spatial relationship of the regional white matter tracts. All figures included in this study are unenhanced in order to closely resemble the white matter fiber tract anatomy encountered in standard laboratory settings.

\section{Results \\ Fiber Dissection}

Upon removing the cortex and superficial U-fibers of the POS, calcarine fissure, cuneus, precuneus, posterior cingulate area, and lingual, parahippocampal, and fusiform gyri, we invariably expose a group of fibers seen to radiate from the area of the posterior precuneus (Brodmann area $[\mathrm{BA}] 7$, anterior bank of POS) to the basal temporooccipital junction (BA37, middle third of fusiform gyrus; Figs. 1 and 2). This distinct white matter bundle, previously described as the $\mathrm{CB}-\mathrm{V}$, follows the trajectory of the POS, bends around the splenium of the corpus callosum, then curves caudomedially passing under the posterior half of the proximal calcarine fissure to reach the posterior parahippocampal area, and further descends under the collateral sulcus in a deeper plane to terminate at the middle third of the fusiform gyrus (BA37, fusiform face area [FFA]; Figs. $3-6)$. In $80 \%$ of cases (12/15 hemispheres), fibers of the CB-V were also noted to terminate at the medial temporal lobe and more specifically at the lateral piriform area (BA35).

This fiber tract follows a dorsomedial to ventrolateral trajectory and usually exhibits three medially projecting "knees," specifically at the level of the FrM, RSC, and parahippocampal gyrus. It displays a triangular silhouette with the narrowest part seen in the area of the posterior precuneus and the widest part in the area of the fusiform gyrus (Figs. 3, 4, and 6). No differences were observed between the left and right hemispheres in terms of the tract's thickness, connectivity pattern, or morphology.

\section{Spatial Relationship of the CB-V With Adjacent Fiber Tracts \\ Inferior Arm of the Cingulum and the CB-V}

Fibers of the CingI originate from the frontal cortices and bend around the splenium of the corpus callosum in an almost parallel trajectory to the midsagittal plane, whereas the $\mathrm{CB}-\mathrm{V}$ fibers originate at the precuneus and run in an oblique dorsolateral to ventromedial direction, crossing the parasagittal plane (Figs. 1-4). Although inferior to the level of the splenium, these two bundles exhibit what is widely known as a "kissing" pattern of fiber distribution. Indeed, the white matter dissection technique allowed us 

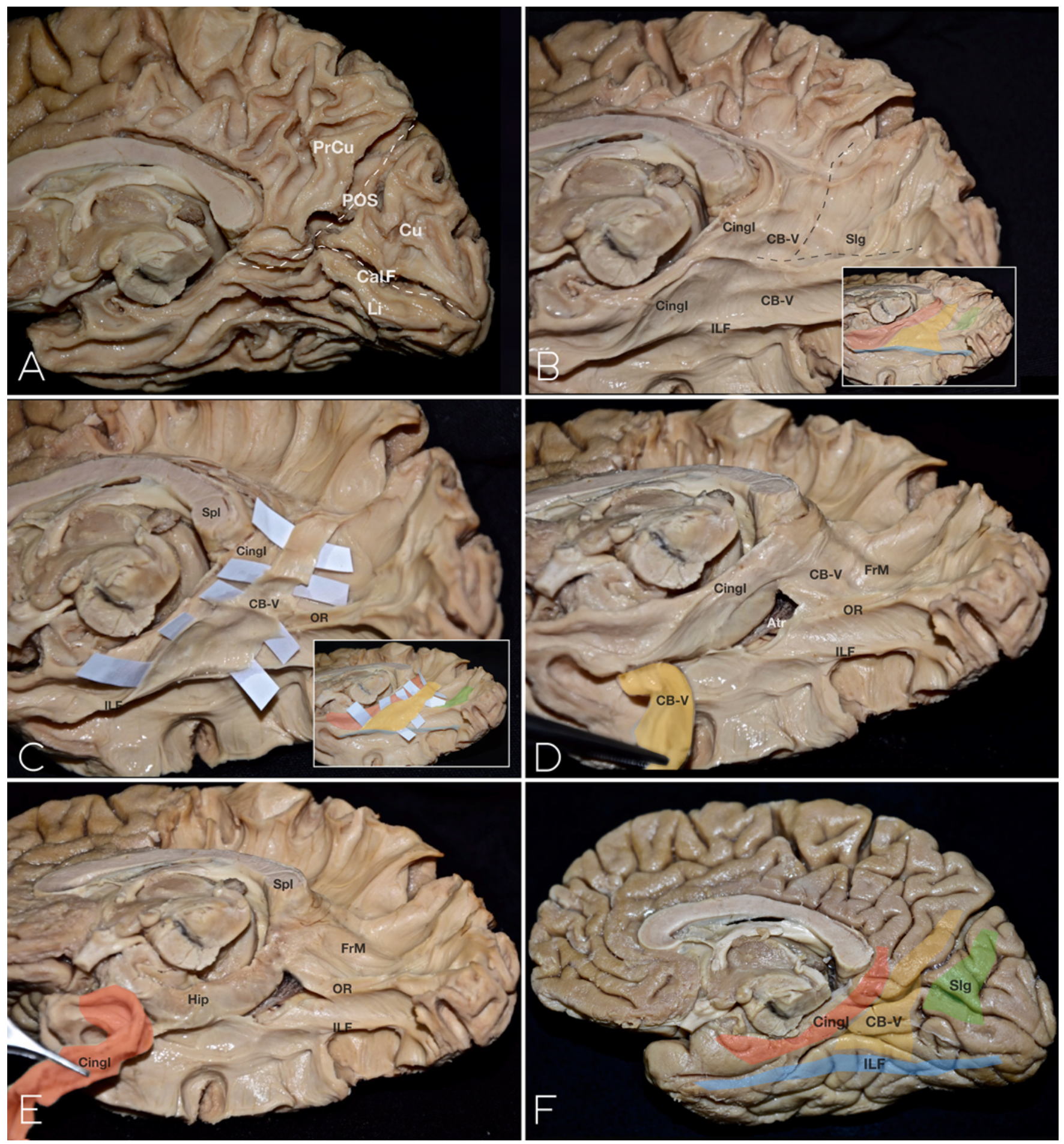

FIG. 1. Progressive dissection of a right hemisphere, medial views. A: Gray matter has been removed. The superficial U-fibers are apparent. The contour of the main sulci is indicated by a dashed white line. B: Upon removing the superficial U-fibers, the inferior arm of the cingulum (Cingl), cingulum bundle V (CB-V), inferior longitudinal fasciculus (ILF), and sledge runner fasciculus (Slg) are visible. These fasciculi are highlighted in red, yellow, blue, and green, respectively (inset, B). C: The fibers of the CB-V are followed along their trajectory, from the posterior precuneus to the area of the fusiform gyrus and medial temporal lobe. The CB-V fibers can be dissected without affecting the structural integrity of the Cingl. These two fiber systems appear in a "kissing" configuration but without intermingling The Cingl, CB-V, Slg, and ILF are highlighted in red, yellow, green, and blue, respectively (inset, C). D: Fibers of the CB-V are followed toward the medial temporal lobe. The structural integrity of the Cingl remains intact. E: The Cingl is gradually dissected. Its inferior terminations follow a trajectory that is medial in relation to that of the CB-V. F: The anatomical silhouettes of the Cingl (red), CB-V (yellow), Slg (green), and ILF (blue) are superimposed on the medial surface anatomy. Atr = atrium; CalF = calcarine fissure; $\mathrm{Cu}=\mathrm{cuneus;}$ FrM = forceps major; $\mathrm{Hip}=$ hippocampus; $\mathrm{Li}=$ lingual gyrus; $\mathrm{OR}=$ optic radiation; $\mathrm{POS}=$ parietooccipital sulcus; $\mathrm{PrCu}=$ precuneus; $\mathrm{Spl}=$ splenium of the corpus callosum. Copyright Christos Koutsarnakis. Published with permission. Figure is available in color online only. 

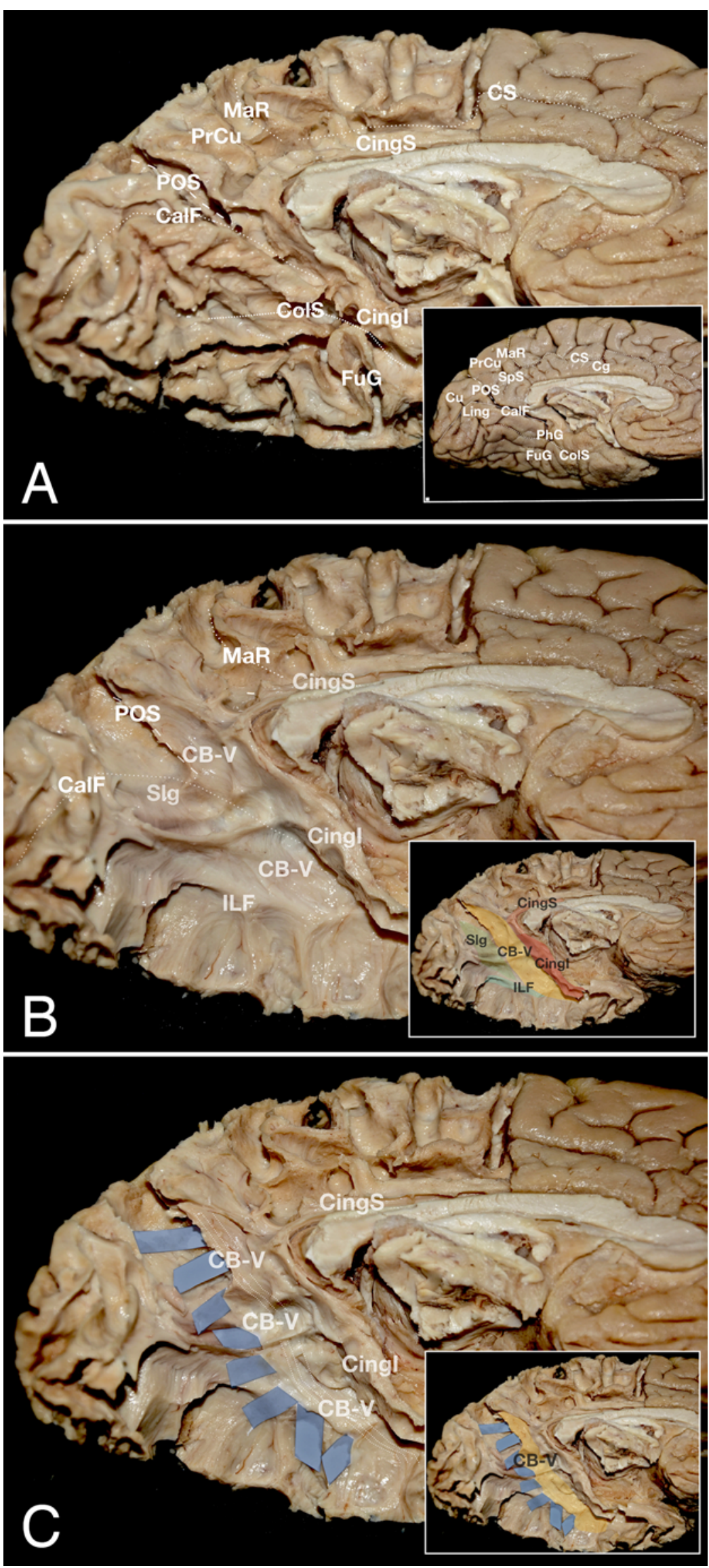

FIG. 2. Progressive dissection of a left hemisphere medial views. A: Superficial U-fibers are exposed. The contours of the main sulci are visible. The superficial landmarks including the sulci and gyri are marked (inset, A). B: U-fibers are removed and the Cingl, CB-V, and Slg are visible. The SIg resides caudally with its fibers running from the anterior cuneus toward the parahippocampal place area (PPA) and retrosplenial cortex (RSC). The CB-V is located between the SIg and Cingl. Its fibers travel anterior to the POS from the posterior precuneus to the middle third of the fusiform gyrus (FuG). Anterior to the $C B-V$, the Cingl projects and terminates at the medial temporal structures. These tracts are highlighted in yellow (CB-V), red (Cingl), and green (Slg; inset, B). C: The $\mathrm{CB}-\mathrm{V}$ is gradually dissected, starting from the posterior precuneus to the FuG. The CB-V and Cingl run as independent pathways. The CB-V is highlighted in yellow (inset, $\mathrm{C}$ ). CalF = calcarine fissure (distal part); $\mathrm{Cg}=$ cingulate gyrus; CingS = superior arm of the cingulum; ColS = collateral sulcus; CS = cingulate sulcus; Ling = lingual gyrus; MaR = marginal ramus of the cingulate sulcus; PhG = parahippocampal gyrus; $\mathrm{SpS}=$ subparietal sulcus See previous figure legend for additional abbreviations. Copyright Christos Koutsarnakis. Published with permission. Figure is available in color online only. 


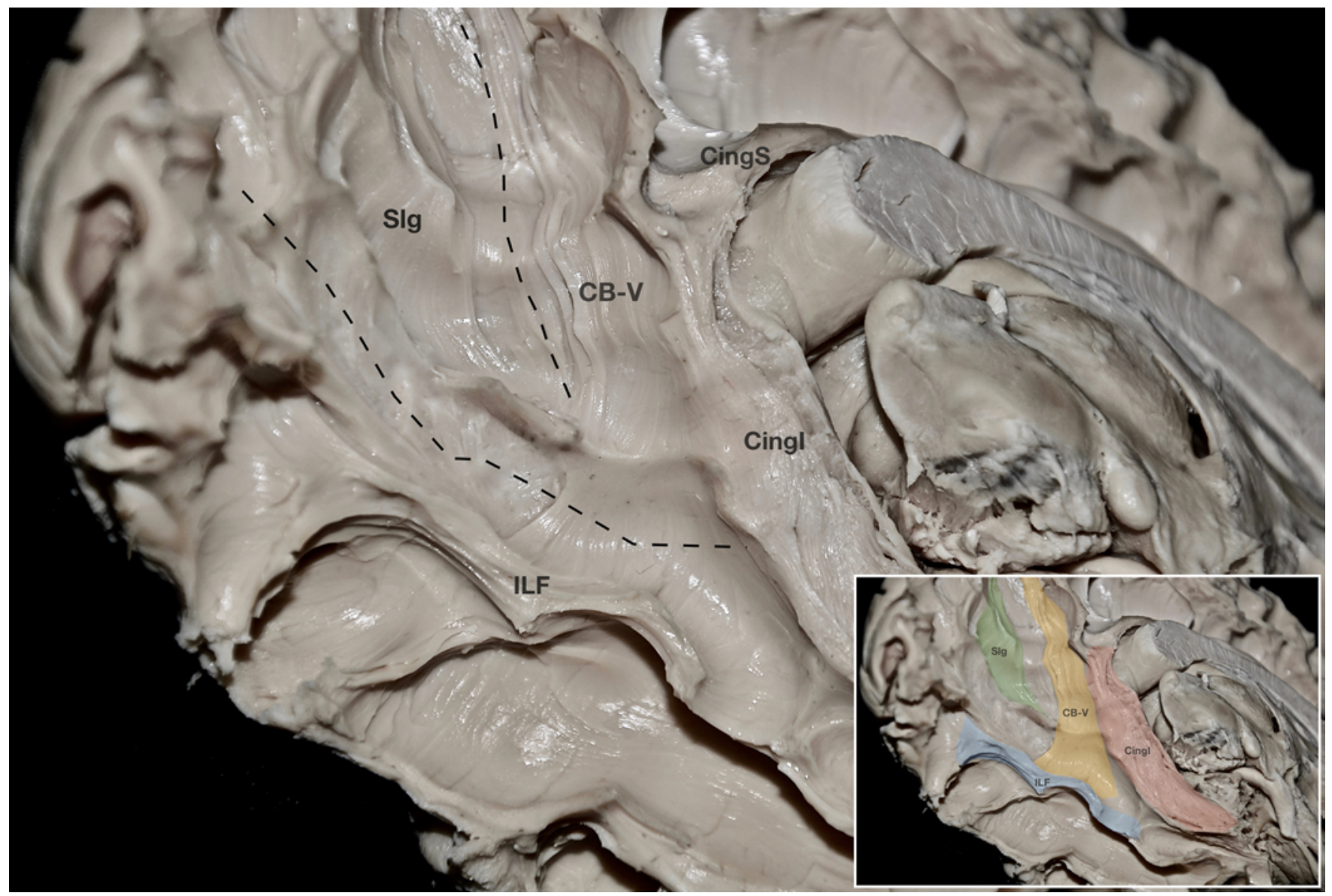

FIG. 3. Left hemisphere, inferomedial view, showing the spatial relationship of the Cingl, ILF, CB-V, and Slg. The Slg follows a short trajectory from the anterior cuneus toward the PPA and RSC and is located caudal to the CB-V. The CB-V is seen to have a longer configuration, originating from the posterior precuneus and traveling mainly toward the FuG. It exhibits a funnel-like shape with its narrowest part in the area of the posterior precuneus and its wider part in the fusiform area. The stem of the ILF travels within the FuG, keeping a trajectory perpendicular to those of the CB-V and Slg. Interestingly, the inferior terminations of the CB-V penetrate the fibers of the ILF to terminate in the midfusiform gyrus. The Cingl curves around the splenium of the corpus callosum to terminate at the medial temporal lobe. The correlative anatomy of the four fasciculi is indicated by red (Cingl), yellow (CB-V), green (Slg), and blue (ILF; inset). See previous figure legends for additional abbreviations. Copyright Christos Koutsarnakis. Published with permission. Figure is available in color online only.

to specifically differentiate the fibers of the CB-V, which are encountered more lateral and caudal in relation to the fibers of the CingI.

\section{Sledge Runner Fasciculus and the CB-V}

We observed the fibers of the cingulum lying adjacent to the fibers of the Slg. This relation was particularly tight with regard to the most anteroinferior part of the Slg, which runs deep to the isthmus of the cingulate gyrus and posterior part of the parahippocampal gyrus.

The Slg is an adjacent fiber tract that also travels in the depth of the POS, in the same plane and in a close spatial relationship with the CB-V (Figs. 1-6). However, in contrast to the $\mathrm{CB}-\mathrm{V}$, which stems from the anterior bank of the POS (caudal precuneus), the Slg originates at the posterior bank of the POS (rostral cuneus) and travels in an oblique rostroventral direction, following a shorter trajectory and terminating at the RSC, parahippocampal place area (PPA), and anterior lingula. The CB-V and Slg fibers are seen to cross the deep segment of the POS and most probably share common terminations in the PPA area (Figs. 1 and 3-5).

\section{Forceps Major and the CB-V}

The FrM, also known as the posterior forceps, consists of fibers originating from the splenium of the corpus callosum and traveling toward the occipital cortex. Compared to fibers of the $\mathrm{CB}-\mathrm{V}$, the fibers of this bundle are seen to reside in a deeper plane and follow a perpendicular trajectory, thus creating a superior knee in the dorsal half of the CB-V (Fig. 1).

\section{Inferior Longitudinal Fasciculus}

The ILF fibers run in the axial plane in the depth of the fusiform gyrus. The CB-V fibers originate from the precuneus and terminate at the midportion of the fusiform gyrus, abutting fibers of the middle third of the ILF in a perpendicular fashion. The $\mathrm{CB}-\mathrm{V}$ fibers that continue to- 


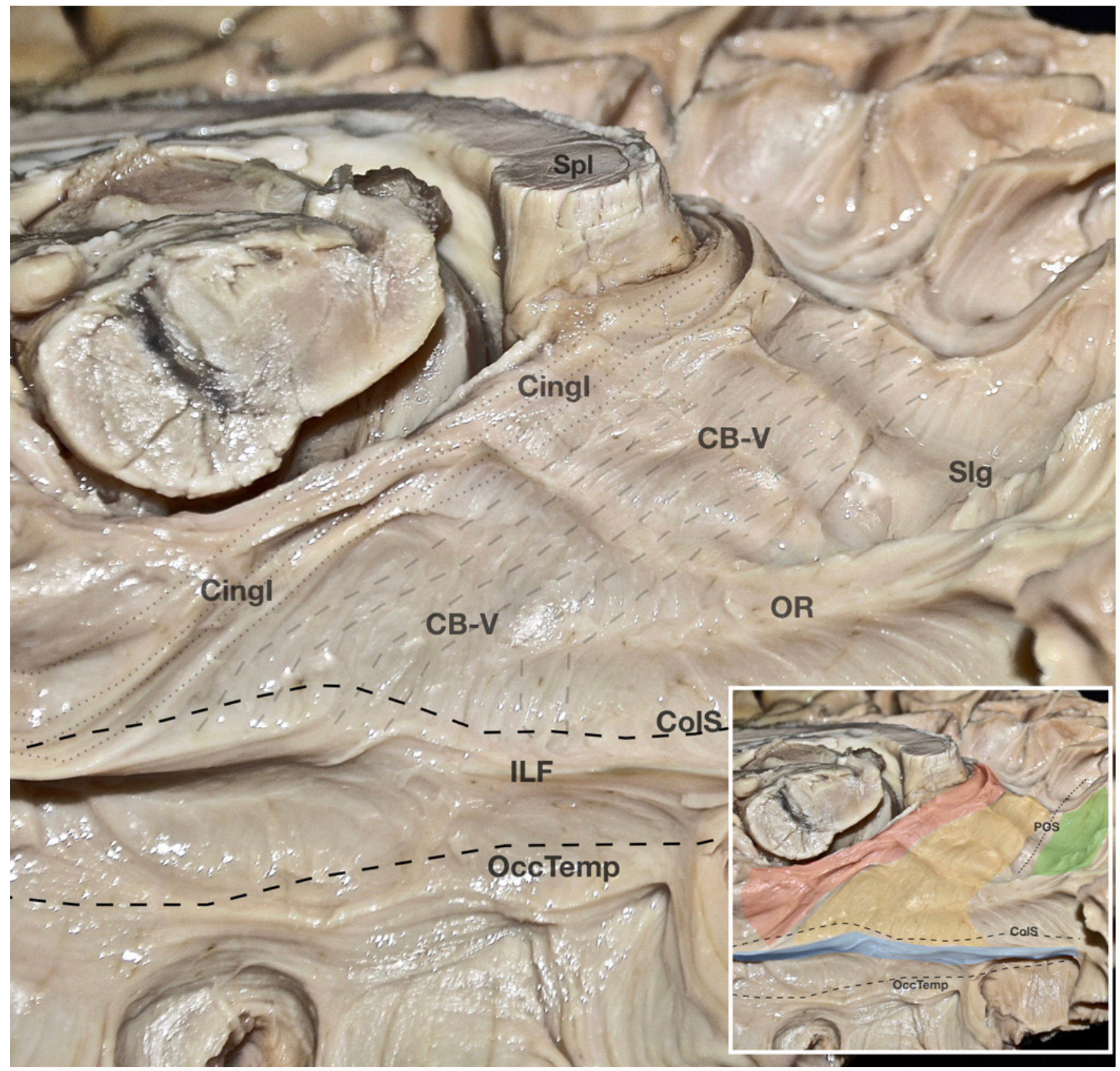

FIG. 4. Right hemisphere, inferomedial view, showing the CB-V and adjacent fasciculi in relation to the sulcal anatomy. The silhouettes of the main sulci, including the collateral sulcus (ColS), occipitotemporal sulcus (OccTemp), and POS, are indicated by gray dots and black dashes. The CB-V (gray dashes) travels anterior to the POS, whereas the Slg (gray dots) lies posteriorly. The ILF is confined within the FuG. The inferiormost fibers of the CB-V travel below the depth of the collateral sulcus terminating in the area of the midfusiform gyrus. The Cingl, CB-V, Slg, and ILF are highlighted with red, yellow, green, and blue, respectively (inset), and the silhouettes of the main sulci are also marked (black dotted and dashed lines) for orientation purposes. See previous figure legends for additional abbreviations. Copyright Christos Koutsarnakis. Published with permission. Figure is available in color online only.

ward the medial temporal pole travel in a direction parallel to that of the ILF (Figs. 4 and 5).

\section{Connectivity}

In all cases, we found $\mathrm{CB}-\mathrm{V}$ fibers terminating in the area of the caudal precuneus (BA7), PPA (BA20), and midfusiform gyrus (BA37). Fibers of the $\mathrm{CB}-\mathrm{V}$ were also noted to terminate in the RSC (BA29 and BA30) in 93\% $(14 / 15)$ of cases. In $80 \%(12 / 15)$ of the specimens, we also encountered fibers of the $\mathrm{CB}-\mathrm{V}$ in the lateral piriform cortex (BA35; Figs. 1, 2, and 5). The connectivity of the CB-V is outlined in Table 1. 


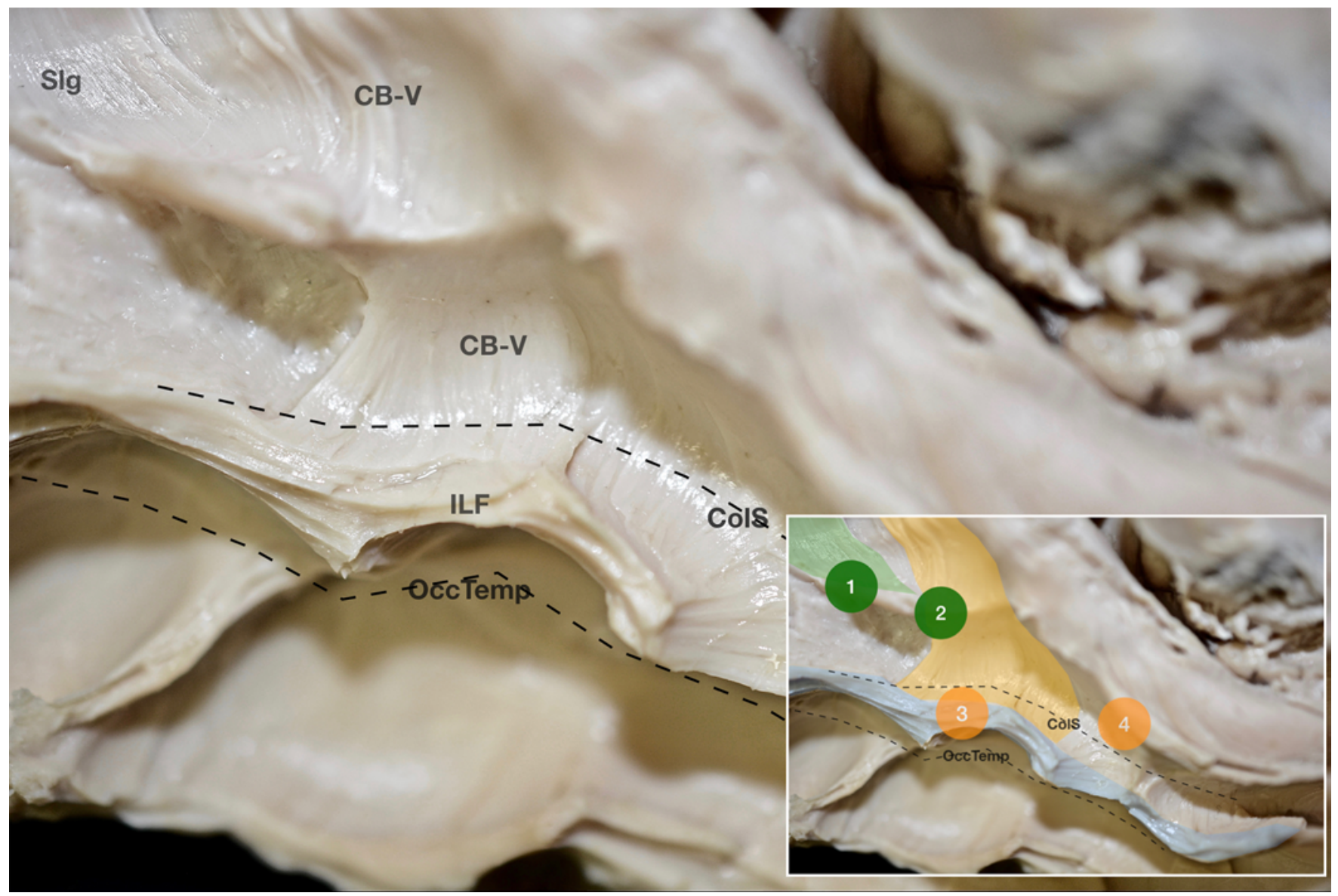

FIG. 5. Close-up of a left hemisphere, inferomedial view. Inferior terminations of the CB-V and SIg are visible. The Slg terminates inferiorly in the RSC and PPA, while the CB-V follows a longer trajectory reaching the level of the FuG. The inferiormost terminations of the Slg and CB-V are highlighted in green and yellow, respectively (inset). The Slg terminates in the area of the RSC (1) and PPA (2), while the CB-V reaches the middle part of the FuG, which corresponds to the fusiform face area (FFA; 3 ) and the medial temporal structures (4). The ILF is highlighted in blue. See previous figure legends for additional abbreviations. Copyright Christos Koutsarnakis. Published with permission. Figure is available in color online only.

\section{Discussion}

The idea of segmentation of major association tracts such as the superior longitudinal fasciculus (SLF) and cingulum has been enthusiastically gaining ground in the field of human brain connectivity as it refines brain circuitry in a more accurate manner. Indeed, several studies focusing on the anatomy of different components of the SLF, ILF, arcuate fasciculus, and cingulum have been recently published. ${ }^{15,20,21}$ According to this concept, these fiber pathways can be studied as individual anatomo-functional entities as they exhibit distinctive connectivity in terms of axonal termination patterns, while their structure and function can be conceptualized as the cluster effect of smaller tributaries that converge and contribute to the formation of a main subcortical "river."

In this context, $\mathrm{Wu}$ and colleagues use the term " $\mathrm{CB}-\mathrm{V}$ " to describe the segment of the cingulum that connects the precuneus with temporobasal and temporomesial structures..$^{15}$ Further, in a study by Jones and colleagues, the same bundle-described as the "parahippocampal cingulum"-was seen to interconnect the posterior precuneus, posterior cingulate cortex, and medial temporal lobe. ${ }^{22}$ To our knowledge, however, the current literature is quite limited on this medially projecting parietotemporal pathway; therefore, its very existence, topography, morphology, and intricate anatomical characteristics have remained vague to date. With this in mind, we strived to provide original, human, ex vivo, direct structural evidence on the existence of the CB-V as a discrete component of the $\mathrm{CB}$ and on the direct axonal connectivity between the precuneal territory and mesiotemporal regions.

Our results demonstrate that the CB-V exhibits extensive cortical projections to the caudal precuneus. Findings in healthy individuals have suggested that the precuneus is the core structure of the DMN. ${ }^{10,23}$ The idea of the DMN was derived from the classic studies of Shulman (1997) and Raichle (2001). ${ }^{24-26}$ These authors, using data stemming from functional imaging (functional MRI and PET), observed a group of cortical areas in the human brain, including the posterior cingulate cortex, medial prefrontal cortex, and precuneus, that were consistently found to exhibit reduced activity during non-self-oriented and goal- 


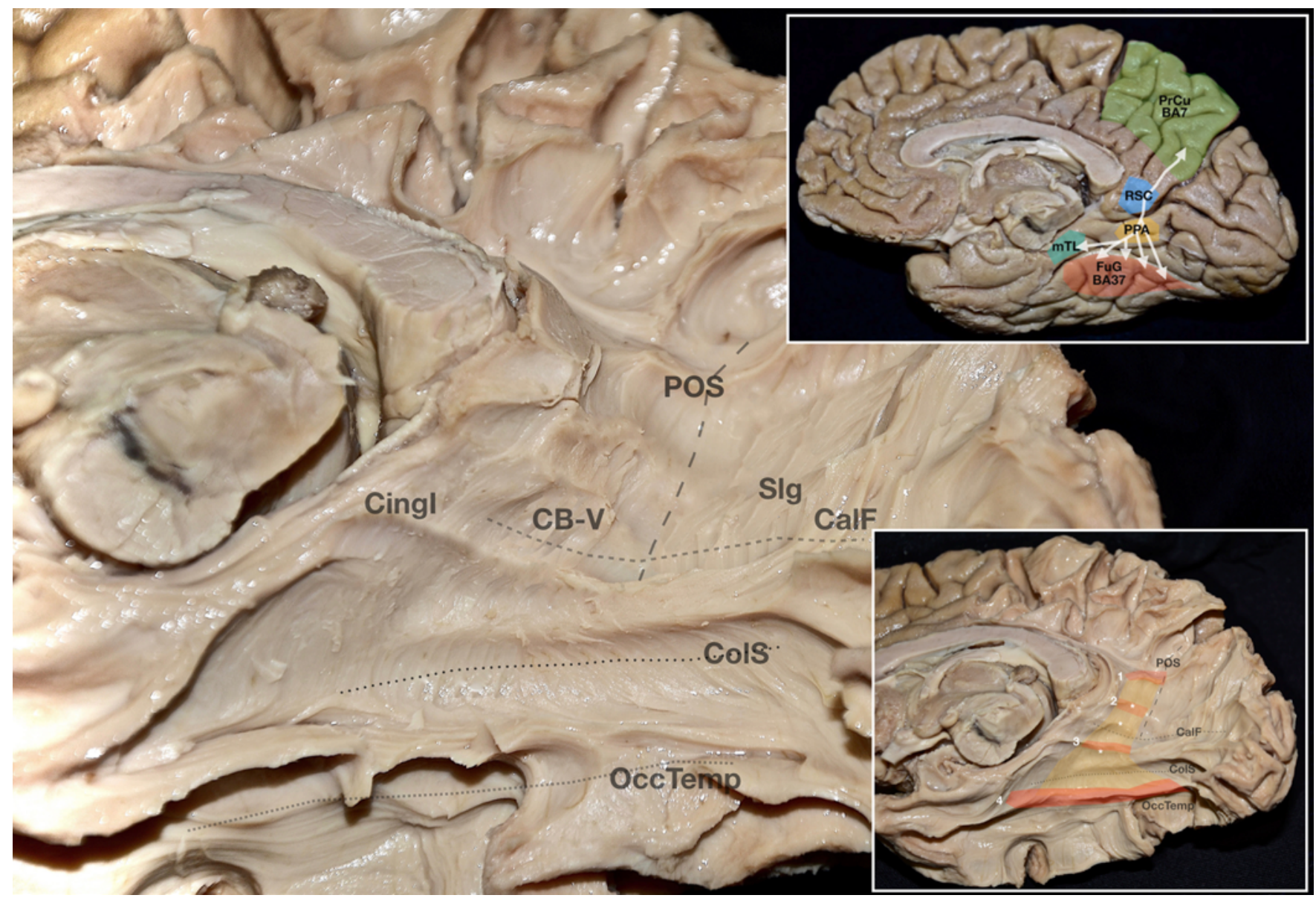

FIG. 6. Inferomedial view of a right hemisphere showing the morphology and connectivity of the CB-V. The CB-V can be seen running at the level of the anterior bank of the POS. Its fibers spread toward the fusiform area and medial temporal lobe. Its narrowest part corresponds to the area of the posterior precuneus, while the widest part corresponds to the area of the FuG. The CB-V exhibits three or four medially projecting "knees," which usually appear near the level of the RSC and PPA. Four termination zones can be appreciated here. The superior terminations of the CB-V project toward the posterior precuneus. Two termination zones are noted at the level of the RSC and PPA. The inferior terminations of the CB-V radiate in a wide area corresponding to the middle and sometimes the posterior aspect of the FuG and part of the pyriform cortex. The trajectory of the CB-V is superimposed on the superficial anatomy (upper inset). Terminations cover a wide area including the posterior precuneus ( $\mathrm{PrCu}$; green), RSC (blue), PPA (yellow), FuG (red), and medial temporal lobe (mTL; teal). The knees of the CB-V are marked with stars (lower inset). The termination zones are highlighted in red: 1 = posterior PrCu; 2 = RSC; $3=$ PPA; 4 = FuG and mTL. See previous figure legends for additional abbreviations. Copyright Christos Koutsarnakis. Published with permission. Figure is available in color online only.

directed tasks. In light of this observation, the term "default mode" was coined by Raichle. ${ }^{6}$ The very same areas show significant coactivation during resting-state conditions and theoretically support a default mode of brain function activated during daydreaming, self-oriented tasks, and theory of mind tasks. This task-negative network allegedly participates in high-order amodal functions such as selfawareness, mind wandering, and future planning..$^{25,27}$

Moreover, the precuneal lobule is consistently found to be activated in a broad range of high-order functions such as navigation, memory retrieval, attention, intelligence comparison, visuospatial imagery, representational similarity analysis, and transitive reasoning. ${ }^{5,28,29}$ In the same vein, data stemming from a recent repetitive transcranial magnetic stimulation study have shown the precuneus to be involved in the process of updating place representations during self-motion. ${ }^{30}$ Additionally, the $\mathrm{CB}-\mathrm{V}$ has been recorded to project to the cortex of the PPA, which is consistently seen to participate in the visuospatial circuitry involving location representation, context retrieval, and perception and utilization of spatial landmarks. ${ }^{4}$

Another key hub area participating in the "navigation pathway" is the RSC, also known as the "retrosplenial complex area." This region encodes visual information and supports navigation, orientation, and spatial memory. ${ }^{4}$ According to our results, the CB-V exhibits its higher fiber density in this brain region. Similarly, and slightly anteriorly, the parahippocampal gyrus is also one of the areas in which the CB-V fibers constantly radiate. This specific region is implicated in high-level visuospatial perception and memory processing, and as recently demonstrated, the underlying structural architecture of these two aforementioned discrete functions is parceled along the longitudinal axis. ${ }^{31}$ More specifically, visuospatial perception 
TABLE 1. Connectivity and putative role of the CB-V

\begin{tabular}{|c|c|c|c|c|c|}
\hline \multirow[b]{2}{*}{ Variable } & \multicolumn{5}{|c|}{ Structure } \\
\hline & Caudal Precuneus & PPA & Midfusiform Gyrus (FFA) & Retrosplenial Cortex & Lateral Piriform Cortex \\
\hline $\mathrm{BA}$ & 7 & 20 & 37 & $29 / 30$ & 35 \\
\hline Percentage & $100 \%$ & $100 \%$ & $100 \%$ & $93 \%$ & $80 \%$ \\
\hline $\begin{array}{l}\text { Possible func- } \\
\text { tional role } 1,3-5,7,23, \\
24,28,31,32\end{array}$ & $\begin{array}{l}\text { Self-related processing, } \\
\text { awareness \& conscious } \\
\text { information, episodic } \\
\text { memory retrieval, vi- } \\
\text { suospatial processing, } \\
\text { attention transitive } \\
\text { reasoning, intelligence } \\
\text { comparison, navigation }\end{array}$ & $\begin{array}{l}\text { Recognition } \\
\text { of places \& } \\
\text { scenes, using } \\
\text { scene informa- } \\
\text { tion to orientate, } \\
\text { location } \\
\text { representation, } \\
\text { context retrieval }\end{array}$ & $\begin{array}{l}\text { Facial recognition } \\
\text { perception, facial ex- } \\
\text { pression, visual mental } \\
\text { imagery, visual word } \\
\text { recognition }\end{array}$ & $\begin{array}{l}\text { Navigation \& orienta- } \\
\text { tion, spatial memory, } \\
\text { planning \& imagining } \\
\text { future events, combin- } \\
\text { ing egocentric \& al- } \\
\text { locentric information, } \\
\text { episodic \& autobio- } \\
\text { graphical memory }\end{array}$ & $\begin{array}{l}\text { Multisensory asso- } \\
\text { ciation, memory, } \\
\text { assessing motiva- } \\
\text { tional significance } \\
\text { of sensory cues, } \\
\text { integration of sensory } \\
\text { cues w/ behavior }\end{array}$ \\
\hline
\end{tabular}

is localized at the posterior half of the parahippocampal gyrus, whereas the anterior half is involved in contextual memory processing. The $\mathrm{CB}-\mathrm{V}$ also exhibits profound cortical projections to the fusiform gyrus and particularly to the FFA. The FFA has been extensively implicated in face recognition, facial expression perception, and visual word recognition, but it has also been reported to participate in a handful of other visual and memory cognitive processes, such as visual mental imagery and visual memory. ${ }^{32}$ The possible functional implications of the $\mathrm{CB}-\mathrm{V}$ are summarized in Table 1.

We have provided direct structural evidence on the existence, topography, morphology, and axonal connectivity of a thus far vague and ambiguous white matter pathway previously known as the " $\mathrm{CB}-\mathrm{V}$ " in the neuroscientific literature. This separate bundle of fibers was noted to participate in the connectivity of high-order cerebral areas such as the caudal precuneus, PPA, RSC, and FFA.

\section{Microsurgical Corridors to Trigonal and Peritrigonal Lesions and Possible Clinical Repercussions With Respect to the CB-V}

The CB-V underlies a wide cerebral area that extends from the precuneus to the fusiform gyrus. This region is exposed and manipulated in an effort to elegantly approach and resect lesions residing in or around the atrium of the lateral ventricle. Trigonal and peritrigonal lesions have been traditionally accessed through a variety of surgical approaches including the posterior transcallosal corridor or the transcortical operative variants through the inferior parietal lobule or posterior temporal lobe. However, the neurological sequelae linked to these surgical trajectories-including disconnection syndromes, damage to the optic radiations, dyslexia, agraphia, apraxia, motor disturbances, and a high incidence of epileptic seizures-have paved the way toward more sophisticated routes in the era of modern microneurosurgery.

In this context, Yasargil was the first to describe the parietooccipital interhemispheric transprecuneus approach for the treatment of peritrigonal lesions. ${ }^{33}$ This surgical avenue was further elaborated by Goel in 1995, who proposed the contralateral interhemispheric transfalcine transprecuneus approach (CITP) to avoid the ill effects of excessive brain retraction. ${ }^{34,35}$ Although elegant, these surgical pathways inevitably involve the manipulation, retraction, or even transgression of the cortex and white matter of the precuneus and neighboring structures. As shown in the current study, the CB-V radiates to this very same area, lying just lateral to the superficial U-fibers of the posterior precuneus and connecting this cortical hub to the PPA, FFA, RSC, and lateral piriform cortex and thus participating in a multimodal association network believed to integrate visuospatial, facial, self-relevant, and other types of cues with mnemonic functions. Interrupting this network could result in disconnection syndromes involving spatial and episodic memory, awareness, and the ability to recognize faces.

The supracerebellar transtentorial transcollateral sulcus (STTS) approach is an alternative sophisticated corridor for accessing atrial and peri-atrial lesions. ${ }^{36}$ Through a wide transsulcal opening of at least $3 \mathrm{~cm}$ of the collateral sulcus, the surgeon can allegedly access trigonal and peritrigonal lesions without damaging the optic radiations. However, doubts have been raised with regard to the visually related morbidity of this approach since the route provided by the collateral sulcus may involve damaging the anterior bundle of the optic radiations. ${ }^{17}$ Additionally, and from a hodotopical point of view, interrupting the inferior part of the $\mathrm{CB}-\mathrm{V}$ within the fusiform gyrus may impede the flow of information arising from BA37 (FFA) relevant to face and word recognition to the precuneal hub.

Limited data exist with regard to memory and supramodal integration deficits following the CITP and STTS approaches. ${ }^{33}$ This could be attributed to the fact that a thorough preoperative and postoperative neuropsychological battery is required to assess deficits in high-order functions subserved by fine tracts such as the $\mathrm{CB}-\mathrm{V}$, something that is not routinely applied in the daily management of such patients. However, in the modern era of extensive multidisciplinary research into the brain connectome, the neuro-oncology surgeon should be an integral part of scientific groups exploring cerebral structure-function relationships not only by providing in vivo data through brain mapping techniques but also by investigating the corticosubcortical architecture through anatomical fiber dissections and diffusion tensor imaging protocols. 


\section{Study Strengths and Limitations}

Reviving the use of the white matter fiber dissection technique in an effort to unveil the structural architecture of brain connectivity has been mainly fueled by the propagation of MRI-based diffusion tensor imaging tractography since indirect tractographic results must be fully validated against a robust and direct anatomical method. Undoubtedly, both tensor and sophisticated nontensor techniques such as high angular resolution diffusion imaging (HARDI), constrained spherical deconvolution (CSD), and global tractography have significantly improved connectome reconstructions and have led to a more accurate in vivo mapping of the cerebral subcortical architecture. Nevertheless, even the newest and most advanced neuroimaging methods generate considerable numbers of false-positive tracts, mainly due to noisy (spurious) peaks or ambiguous fanning and bending fiber populations. ${ }^{37-40}$ This effect is further accentuated when long-distance connections are explored, as the reconstruction error accumulates with each tracking step. ${ }^{37,40,41}$ Therefore, the current literature has emphasized that the fundamental problem tractography faces is that it infers connectivity from local orientation fields, and even in ideal experimental conditions, the anatomical accuracy of an indirect method like diffusion-weighted imaging is suboptimal. ${ }^{37-41}$ For these reasons, the application of a direct anatomical method such as the white matter dissection technique in validating tractographic results and, most importantly, in ruling out invalid and erroneous tracts is mandatory.

The fiber dissection method (freeze-thaw process) has been microscopically proven to maintain the anatomical integrity of the white matter fibers, thus providing structural evidence of high accuracy. ${ }^{42}$ Further, the development of the "cortex-sparing" white matter dissection approach has allowed for a more detailed and comprehensive investigation of the 3D cerebral connectivity and termination pattern of fiber pathways. ${ }^{43}$ Thus, this technique is currently regarded as the "gold-standard" procedure for validating tractographic data; therefore, we have been intentionally using it as our basic method to explore the subcortical architecture of complex brain territories and novel or underrecognized fiber tracts. ${ }^{16,18,44}$

This technique, apart from being an in vitro, time-consuming, operator-dependent, and very expensive process, also provides data of lower spatial resolution in comparison to that provided by optical coherence tomography. ${ }^{45}$ Last, in our experience, the sensitivity of this method can be relatively reduced when investigating fiber tracts that cross in a perpendicular trajectory since proper dissection of the one involves disfigurement of the other.

Studying the subcortical architecture and connectivity through the fiber dissection technique is not just a simple, academic laboratory exercise that improves a static view of brain anatomy and sharpens neurosurgical hand skills. Instead, this endeavor provides the neurosurgeon with a more accurate understanding of brain structure-function relationships and subsequently informs surgical practice not only in the context of modern brain mapping techniques, which aim to optimize the patient's onco-functional balance, but also in the process of preoperative informed consent and approach-related decision-making.
Modern neurosurgery is and should remain an integral part of a wider multidisciplinary approach aiming to gain better insight into cerebral anatomo-functional connectivity - not only by providing awake online data about cognitive behaviors but also by investigating the cortico-subcortical architecture through anatomical fiber dissections and diffusion tensor imaging protocols.

\section{Conclusions}

Through the fiber microdissection technique, we have provided data supporting the existence of the $\mathrm{CB}-\mathrm{V}$ as a discrete subcomponent of the cingulum pathway. We also provided solid structural background on the direct axonal connectivity of the precuneus and medial temporal lobe, cerebral territories that are heavily implicated in the neural circuit of core cognitive functions such as face and word recognition, facial expression perception, spatial navigation and updating, visuospatial perception memory, and imagery. This constant anatomo-functional "dialogue" between these regions through discrete white matter pathways provides useful insights into the adjustment and integration of the neural inputs and correlates of complex social cognition. Last, we have also raised awareness with regard to the involvement of the $\mathrm{CB}-\mathrm{V}$ in microneurosurgical corridors employed to access lesions residing in or around the atrium of the lateral ventricle.

\section{References}

1. Hebscher M, Levine B, Gilboa A. The precuneus and hippocampus contribute to individual differences in the unfolding of spatial representations during episodic autobiographical memory. Neuropsychologia. 2018;110:123-133.

2. Mancini M, Mastropasqua C, Bonnì S, et al. Theta burst stimulation of the precuneus modulates resting state connectivity in the left temporal pole. Brain Topogr. 2017;30(3):312319.

3. Burles F, Slone E, Iaria G. Dorso-medial and ventro-lateral functional specialization of the human retrosplenial complex in spatial updating and orienting. Brain Struct Funct. 2017;222(3):1481-1493.

4. Epstein RA, Patai EZ, Julian JB, Spiers HJ. The cognitive map in humans: spatial navigation and beyond. Nat Neurosci. 2017;20(11):1504-1513.

5. Marchette SA, Vass LK, Ryan J, Epstein RA. Anchoring the neural compass: coding of local spatial reference frames in human medial parietal lobe. Nat Neurosci. 2014;17(11):15981606.

6. Raichle ME. The brain's default mode network. Annu Rev Neurosci. 2015;38:433-447.

7. Sheldon S, Farb N, Palombo DJ, Levine B. Intrinsic medial temporal lobe connectivity relates to individual differences in episodic autobiographical remembering. Cortex. 2016;74:206-216.

8. Sun J, Liu Z, Rolls ET, et al. Verbal creativity correlates with the temporal variability of brain networks during the resting state. Cereb Cortex. 2019;29(3):1047-1058.

9. Tosoni A, Pitzalis S, Committeri G, et al. Resting-state connectivity and functional specialization in human medial parieto-occipital cortex. Brain Struct Funct. 2015;220(6):33073321.

10. Utevsky AV, Smith DV, Huettel SA. Precuneus is a functional core of the default-mode network. J Neurosci. 2014;34(3):932-940.

11. Zhang J, Andreano JM, Dickerson BC, et al. Stronger func- 
tional connectivity in the default mode and salience networks is associated with youthful memory in superaging. Cereb Cortex. 2020;30(1):72-84.

12. Liu Z, Zhang J, Zhang K, et al. Distinguishable brain networks relate disease susceptibility to symptom expression in schizophrenia. Hum Brain Mapp. 2018;39(9):3503-3515.

13. Wang Z, Williams VJ, Stephens KA, et al. The effect of white matter signal abnormalities on default mode network connectivity in mild cognitive impairment. Hum Brain Mapp. 2020;41(5):1237-1248.

14. Zhu Y, Tang Y, Zhang T, et al. Reduced functional connectivity between bilateral precuneus and contralateral parahippocampus in schizotypal personality disorder. BMC Psychiatry. 2017;17(1):48.

15. Wu Y, Sun D, Wang Y, et al. Segmentation of the cingulum bundle in the human brain: a new perspective based on DSI tractography and fiber dissection study. Front Neuroanat. 2016;10:84.

16. Komaitis S, Kalyvas AV, Skandalakis GP, et al. The frontal longitudinal system as revealed through the fiber microdissection technique: structural evidence underpinning the direct connectivity of the prefrontal-premotor circuitry [published online October 4, 2019]. J Neurosurg. doi:10.3171/2019.6.JNS191224

17. Koutsarnakis C, Kalyvas AV, Komaitis S, et al. Defining the relationship of the optic radiation to the roof and floor of the ventricular atrium: a focused microanatomical study. $\mathrm{J} \mathrm{Neu-}$ rosurg. 2019;130(5):1728-1739.

18. Koutsarnakis C, Kalyvas AV, Skandalakis GP, et al. Sledge runner fasciculus: anatomic architecture and tractographic morphology. Brain Struct Funct. 2019;224(3):1051-1066.

19. Koutsarnakis C, Liakos F, Kalyvas AV, et al. Approaching the atrium through the intraparietal sulcus: mapping the sulcal morphology and correlating the surgical corridor to underlying fiber tracts. Oper Neurosurg (Hagerstown). 2017;13(4):503-516.

20. Panesar SS, Belo JTA, Yeh FC, Fernandez-Miranda JC. Structure, asymmetry, and connectivity of the human temporo-parietal aslant and vertical occipital fasciculi. Brain Struct Funct. 2019;224(2):907-923.

21. Panesar SS, Yeh FC, Jacquesson T, et al. A quantitative tractography study into the connectivity, segmentation and laterality of the human inferior longitudinal fasciculus. Front Neuroanat. 2018;12:47.

22. Jones DK, Christiansen KF, Chapman RJ, Aggleton JP. Distinct subdivisions of the cingulum bundle revealed by diffusion MRI fibre tracking: implications for neuropsychological investigations. Neuropsychologia. 2013;51(1):67-78.

23. Fransson P, Marrelec G. The precuneus/posterior cingulate cortex plays a pivotal role in the default mode network: Evidence from a partial correlation network analysis. Neuroimage. 2008;42(3):1178-1184.

24. Cavanna AE, Trimble MR. The precuneus: a review of its functional anatomy and behavioural correlates. Brain. 2006;129(Pt 3):564-583.

25. Greicius MD, Krasnow B, Reiss AL, Menon V. Functional connectivity in the resting brain: a network analysis of the default mode hypothesis. Proc Natl Acad Sci U S A. 2003;100(1):253-258.

26. Raichle ME, MacLeod AM, Snyder AZ, et al. A default mode of brain function. Proc Natl Acad Sci U S A. 2001;98(2):676-682.

27. Vatansever D, Menon DK, Manktelow AE, et al. Default mode network connectivity during task execution. Neuroimage. 2015;122:96-104.

28. Paladini RE, Müri RM, Meichtry J, et al. The influence of alertness on the spatial deployment of visual attention is mediated by the excitability of the posterior parietal cortices. Cereb Cortex. 2017;27(1):233-243.
29. Rosen ML, Stern CE, Devaney KJ, Somers DC. Cortical and subcortical contributions to long-term memory-guided visuospatial attention. Cereb Cortex. 2018;28(8):2935-2947.

30. Müller NG, Riemer M, Brandt L, Wolbers T. Repetitive transcranial magnetic stimulation reveals a causal role of the human precuneus in spatial updating. Sci Rep. 2018;8(1):10171.

31. Baumann O, Mattingley JB. Functional organization of the parahippocampal cortex: dissociable roles for context representations and the perception of visual scenes. J Neurosci. 2016;36(8):2536-2542.

32. Harvey DY, Burgund ED. Neural adaptation across viewpoint and exemplar in fusiform cortex. Brain Cogn. 2012;80(1):33-44.

33. Tokunaga K, Tamiya T, Date I. Transient memory disturbance after removal of an intraventricular trigonal meningioma by a parieto-occipital interhemispheric precuneus approach: case report. Surg Neurol. 2006;65(2):167-169.

34. Bohnstedt BN, Kulwin CG, Shah MV, Cohen-Gadol AA. Posterior interhemispheric transfalcine transprecuneus approach for microsurgical resection of periatrial lesions: indications, technique, and outcomes. J Neurosurg. 2015; 123(4):1045-1054.

35. Xie T, Sun C, Zhang X, et al. The contralateral transfalcine transprecuneus approach to the atrium of the lateral ventricle: operative technique and surgical results. Neurosurgery. 2015;11(suppl 2):110-118.

36. Zhao X, Borba Moreira L, Cavallo C, et al. Quantitative endoscopic comparison of contralateral interhemispheric transprecuneus and supracerebellar transtentorial transcollateral sulcus approaches to the atrium. World Neurosurg. 2019;122:e215-e225.

37. Maier-Hein KH, Neher PF, Houde JC, et al. The challenge of mapping the human connectome based on diffusion tractography. Nat Commun. 2017;8(1):1349.

38. Reveley C, Seth AK, Pierpaoli C, et al. Superficial white matter fiber systems impede detection of long-range cortical connections in diffusion MR tractography. Proc Natl Acad Sci U S A. 2015;112(21):E2820-E2828.

39. Schilling K, Gao Y, Janve V, et al. Confirmation of a gyral bias in diffusion MRI fiber tractography. Hum Brain Mapp. 2018;39(3):1449-1466.

40. Sinke MRT, Otte WM, Christiaens D, et al. Diffusion MRIbased cortical connectome reconstruction: dependency on tractography procedures and neuroanatomical characteristics. Brain Struct Funct. 2018;223(5):2269-2285.

41. Thomas C, Ye FQ, Irfanoglu MO, et al. Anatomical accuracy of brain connections derived from diffusion MRI tractography is inherently limited. Proc Natl Acad Sci U S A. 2014;111(46):16574-16579.

42. Zemmoura I, Blanchard E, Raynal PI, et al. How Klingler's dissection permits exploration of brain structural connectivity? An electron microscopy study of human white matter. Brain Struct Funct. 2016;221(5):2477-2486.

43. Martino J, De Witt Hamer PC, Vergani F, et al. Cortexsparing fiber dissection: an improved method for the study of white matter anatomy in the human brain. J Anat. 2011;219(4):531-541.

44. Liakos F, Koutsarnakis C. The role of white matter dissection technique in modern neuroimaging: can neuroradiologists benefit from its use? Surg Radiol Anat. 2016;38(2):275-276.

45. Wang H, Black AJ, Zhu J, et al. Reconstructing micrometerscale fiber pathways in the brain: multi-contrast optical coherence tomography based tractography. Neuroimage. 2011;58(4):984-992.

\section{Disclosures}

Dr. Hadjipanayis is a consultant for Synaptive and receives royalties from NX Development Corp. 


\section{Author Contributions}

Conception and design: Koutsarnakis, Skandalakis, Komaitis, Kalyvas. Acquisition of data: Koutsarnakis, Skandalakis,

Komaitis, Kalyvas, Lani, Kontrafouri, Drosos, Fountas, Kapsalaki.

Analysis and interpretation of data: Koutsarnakis, Skandalakis,

Komaitis, Kalyvas, Lani, Kontrafouri, Liakos, Piagkou,

Placantonakis, Golfinos, Fountas, Kapsalaki, Hadjipanayis,

Stranjalis. Drafting the article: Koutsarnakis, Skandalakis,

Komaitis, Kapsalaki. Critically revising the article: Koutsarnakis,

Skandalakis, Komaitis, Kalyvas, Placantonakis, Hadjipanayis,

Stranjalis. Reviewed submitted version of manuscript: all

authors. Approved the final version of the manuscript on behalf

of all authors: Koutsarnakis. Administrative/technical/material

support: Stranjalis. Study supervision: Koutsarnakis, Skandalakis, Komaitis, Kalyvas, Piagkou, Placantonakis, Golfinos, Fountas,

Kapsalaki, Hadjipanayis, Stranjalis.

\section{Correspondence}

Christos Koutsarnakis: Evangelismos Hospital, National and Kapodistrian University of Athens, Greece.ckouts@hotmail.co.uk. 